\title{
ACCIDENTES EN LA EDAD ESCOLAR
}

\author{
Drs, ERNESTO MEDINA y ANA MARLA KAEMPFT'ER
}

\author{
Cáredra de Higiene y Medicina Perpentiva dé Prof. Hernán Romero. Universidad de Chile.
}

Los accidentes constituyen un motivo de preocupación creciente a lo largo del mundo tanto por el número creciente de muertes, lesión, invalidez y pérdida económica, como por su predilección por la gente joven: en el momento actual, $y$ casi sin que nos hayamos percatado de su importancia, representan ya la primera causa de muerte en la población chilena entre 10 y 44 años.

En los países de alto nivel de vida, los esfuerzos sistemáticos de protección de la salud han determinado una sustancial reducción de aquellos problemas susceptibles de prevención fácil. El descenso de la mortalidad infantil y de la mortalidad por enfermedades infecciosas ha traido sin embargo, como contrapartida, el que la patología más difícil de controlar haya adquirido una importancia proporcional mayor, y en el momento actual, una buena parte del mundo soporta una pesada carga de enfermedades cardiovasculares, tumores y accidentes. En el caso particular de éstos, las características propias de la vida moderna y la concentración de la población en ciudades han colaborado en forma efectiva para hacer de ellos un riesgo de gran magnitud.

En nuestro país se da la condición paradojal de que, pese a la persistencia de un grave problema de mortalidad infantil $y$ por infecciosas, los accidentes han adquirido un gran volumen. En efecto, la información disponitble muestra a Chile con la mortalidad más alta del mundo por causas violentas entre 32 países que publican datos de calidad; se producen anualmente unas 6.000 defunciones que corresponden a un $7 \%$ del total y a una tasa aproximada de 80 por 100.000 habitantes; solamente el Servicio Nacional de Salud necesita ingresar cada año a sus establecimientos 37.000 accidentados suficientemente graves como para requerir internación, equivalentes a un $7 \%$ de las hospitalizaciones totales de esa institución y a un $20 \%$ de las masculinas; un cálculo estimativo prudencial sugiere la producción de 370.000 lesiones anuales; finatmente, dada la peculiar distribución por edades de los accidentes, ellos constituyen una de los mecanismos más importantes de invalideces de larga duración y de pérdida de años por vivir. Algunos problemas adquieren especial realce. Iras muertes por accidentes del tránsito, aproximadamente 2.000 anuales, nos colocan entre los países de máxima mortalidad por esta causa y en todo caso tenemos el dudoso privilegio de ostentar la mortalidad de peatones más alta del mundo. Los accidentes del trabajo que ocasionan uno o más días de invalidez suman 125.000 al año y su costo global representa para cada habitante un desembolso anual cercano a los $10 \mathrm{E}$ que se agregan al costo de producción y que pagamos todos los chilenos. En el grupo de edad escolar son responsables de 499 muertes anuales en 1959 ( $21 \%$ del total) lo que les da la primera prioridad como causa de muerte después de enfermedades respiratorias y sitúa a Chile en los primeros Iugares de América en cuanto a riesgo infantil de accidentes. La situación es todavía peor para el grupo preescolar en el que las tasas de muerte por violencia son las más altas del Continente.

Los accidentes se revelan así como un problema considerable y que no muestra indicio alguno hacia el decrecimiento. $\mathrm{Si}$ apareciera una enfermedad epidémica que determina diariamente 1.000 casos y 20 muertes como ocurre con la violencia, se proveerían sin duda recursos extraordinarios, pero el carácter endémico de ella y el desconocimiento de su volumen y trascendencia han determinado sólo en forma reciente, el que diversos grupos de gente interesada haya comenzado a agitar el ambiente en un afán de crear conciencia de su existencia y en el convencimiento que el volumen del riesgo no permite seguir retardando la aplicación de medidas de control que la experiencia extranjera ha mostrado como efectivas. 
Como se comprende el primer paso a utilizar en la prevención, reside en el conocimiento adecuado del fenómeno $\mathrm{y}$ en ese sentido diversos autores ${ }^{1}{ }^{2}{ }_{-} 3_{-} t_{-} 0_{-} 7_{-} 10_{-} 11_{-}$ $12_{1} 1 ;$ han comunicado en nuestro medio diferentes tipos de experiencia o investigación epidemiológica. Esta rama de la medicina, concebida injcialmente para el estudio de las enfermedades infecciosas ha desarrollado técnicas de análisis de la enfermedad como fenómeno colectivo, $y$ su aplicación al campo de los accidentes ha mostrado que ellos siguen las mismas leyes generales y tienen una conducta similar a la de las enfermedades. Así, constituyen un problema endémico, permanente, sobre la colectividad; puede estimarse un número de casos esperados; aparecen estallidos epidémicos y una clara variación geográfica y estacional, y factores individuales claramente definidos como riesgo diverso según edad, sexo u otras características de la población. Su estudio permite conocer la importancia de diversos elementos causales e influir en los eslabones débiles de la cadena de producción ${ }^{2}$.

La investigación en torno a accidentes ha sido profusa en los últimos tiempos $\mathrm{y}$ ha estado basada fundamentalmente en el interés despertado en torno al derroche económico que ellos significan $\mathrm{y}$ a la ventajosa inversión que representa la seguridad en el costo total de la violencia. Probablemente el hallazgo mayor, el aporte más definido que aquella ha entregado es que los accidentes no son realmente accidentales. Hay clara demostración de que ellos no son fortuitos, no se producen porque sí, por mala suerte o porque son inevitables. A la inversa, la mayoría tienen causas perfectamente definidas $y$ susceptibles de modificar; hay una cadena de eventos que llevan más o menos fatalmente a la producción de determinados accidentes. Como se comprende, si los concibiéramos como hechos incontrolables y debidos al azar las posibilidades de prevención serían nulas.

Los accidentes pueden ser interpretados como un resultado de la interacción entre el hombre y su ambiente y el estudio de estas interacciones suministra las bases de prevención. Ellos representan un excelente ejemplo de causalidad múltiple en la que es posible separar por lo menos tres tipos de elementos: agente, mesonero, y ambiente. Pero, mientras la importancia del ambiente es obvia y el agente nos parece igualmente claro, no surge con tanta nitidez la importancia del tercer elemento, aún cuando si se examina críticamente, la mayoría de los accidentes se relacionan más estrechamente con características individuales que con otro tipo de factores. En la constelación generadora de accidentes aparecen así, factores ambientales que pueden ser resumidos en situaciones $y$ condiciones riesgosas, y factores individuales de mayor susceptibilidad.

En nuestro país, la investigación ha abordado los aspectos generales ${ }^{2,11} 12$, los problemas del tránsito ${ }^{1}$ y del trabajo ${ }^{3}$ i-i_s_10 y últimamente la morbilidad atendida en servicios de urgencia ${ }^{4}{ }^{13}$. Pero hasta este momento no se ha utilizado la población escolar como fuente de datos. Los resultados de este enfoque se presentan en esta comunicación.

\section{MIATERIAL Y MÉTODO}

La investigación se llevó a cabo en 31 escuelas primarias del $7^{\circ}$ Sector Escolar de Santiago (Quinta Normal y Barrancas) con un total de 15.638 alumnos matriculados y una asistencia media mensual de 14.099 alumnos. Se solicitó y obtuvo la colaboración de los directores y cada profesor a cargo de un curso se hizo responsable del llene de un formulario frente a cualquier tipo de accidente sufrido por sus alumnos. La ficha registraba los datos básicos de identificación i nombre, edad, sexo, escuela, curso), sitio, circunstancias y características del accidente, lesiones producidas, atención prestada y pérdida de clase. La pesquisa fué efectuada durante los meses de Octubre y Noviembre de 1959.

\section{RESULTADOS}

Con Ia técnica empleada se registraron en el período en estudio un total de 636 accidentes, equivalentes a una tasa mensual de incidencia de 22,5 por 1.000 alumnos. La información obtenida permite delinear algunas de Ias características fundamentales de Ios accidentes en la época escolar. 
ACCIDENTES EN LA EDAD ESCOLAR. - Dr. Ennesto Medina y col.

TABLA NO 1

FRECUENOLA DE ACCIDENTES SEGUN CURSOS $Y$ SEXO. TASAS MIENSDALES POR 1.000 ALUMNOS.

\begin{tabular}{|c|c|c|c|c|c|c|}
\hline \multirow{2}{*}{ Curso } & \multicolumn{2}{|c|}{ Hombres } & \multicolumn{2}{|c|}{ Mujeres } & \multicolumn{2}{|c|}{$T O T A L$} \\
\hline & No de alumnos & Tasa & Nọ de sllummos & Tass & No de filumos & Tasa \\
\hline $\begin{array}{c}\text { Porpulario } \\
I \\
\text { II } \\
\text { III } \\
\text { IV } \\
\text { VI } \\
\text { yI } \\
\text { Pocaclonal }\end{array}$ & $\begin{array}{r}112 \\
1.344 \\
1.237 \\
1.105 \\
1.006 \\
798 \\
517 \\
.251\end{array}$ & $\begin{array}{l}75,8 \\
30,4 \\
26,6 \\
21,7 \\
27,3 \\
35,7 \\
42,5 \\
35,8\end{array}$ & $\begin{array}{r}101 \\
1.841 \\
1.577 \\
1.342 \\
1.131 \\
972 \\
585 \\
-\end{array}$ & $\begin{array}{r}39,6 \\
8,7 \\
19,9 \\
20,5 \\
21,7 \\
19,0 \\
21,3 \\
2\end{array}$ & $\begin{array}{r}213 \\
3.395 \\
2.794 \\
2.447 \\
2.137 \\
1.770 \\
1.102 \\
251\end{array}$ & $\begin{array}{l}58,7 \\
14,0 \\
22,9 \\
21,0 \\
24,3 \\
26,5 \\
31,3 \\
35,8\end{array}$ \\
\hline TOTAL & 6.570 & 28,8 & 7.529 & 17,7 & 14.099 & 22,5 \\
\hline
\end{tabular}

Se observó en primer término una clara diferenciación según sexo (Tabla Ni 1). El riesgo masculino aparece como un $60 \%$ superior al femenino y la diferencia entre las tasas respectivas $(28,8 \mathrm{y}$ 17,7 por 1.000 alumnos) es estadísticamente significativa, correspondiendo a una probabilidad de azar inferior al uno por mil.

La mayor frecuencia o el mayor xiesgo masculino en el problema de accidentes es concordante con la experiencia mundial y nacional y depende de la especial sicología del varón. En nuestra investigación todos los tipos de accidentes son dos veces más frecuentes en el hombre con la excepción de los domiciliarios, situación que depende de la importante colaboración de las niñas en las actividades del hogar. Los masculinos son igualmente más graves si se les juzga a través de la asistencia recibida. El mayor riesgo masculino es especialmente destacado para las lesiones sufridas en cara y cabeza.

La probabilidad de accidentes aumenta con la edad elevándose de 14 por 1.000 en aquellos pertenecientes al primer año primario, a 31,3 en los del $6^{\circ}$ curso. Hacen excepción a esta situación general los datos provenientes de las escuelas de párvulos cuya alta frecuencia probablemente dependa más que de un hecho real, del estrecho contacto de la profesora con sus alumnos y consecuentemente un mejor registro. El aumento del riesgo con la edad se observa en ambos sexos. Llama la atención que el incremento de aquella se acompaña de mayor frecuencia de accidentes del tránsito y de una mayor gravedad; mientras en las edades bajas la mitad requiere asistencia profesional, dicha proporción sube a dos tercios en edades mayores. El aumento de edad representa igualmente una disminución de las lesiones cefálicas con aumento proporcional de lesiones de los miembros.

Los accidentes registrados ocurrieron preferentemente en las escuelas $(39,8 \%$ del total) y sitios públicos $(31,3 \%)$. En una posición intermedia aparecen los domiciliarios $(21,0 \%)$ siendo mucho menos frecuentes los accidentes del tránsito $(7,4$; $)$ y excepcionales, aquellos que el escolar sufrió por razones de trabajo $(0,5 \%)$. Los accidentes ocurridos en la escuela aparecen con alta frecuencia $y$ menor gravedad que el resto siendo posible que el hecho dependa sólo de un mejor conocimeinto. Los otros tipos son relativamente comparables en cuanto a gravedad, medida a través de índices tales como la proporción de accidentes graves, porcentaje con atención profesional o pérdida de clases superiores a $\boldsymbol{B}$ días.

El estudio de las circunstancias que operaban en el momento de producción (Tabla No 2) muestra como los juegos infantiles representan una actividad relativamente riesgosa: casi la mitad de los accidentes registrados ocurrieron mientras el niño se encontraba jugando.

El tránsito por la vía pública, actividades escolares diversas $y$ situaciones in- 
TABLA NOQ 2

OIRGUNSTANCLA DE PRODUCCTON DE ACCIDENTTES

\begin{tabular}{|c|c|c|}
\hline Circunstancia & NQ & $\%$ \\
\hline $\begin{array}{l}\text { Juego en la escuela } \\
\text { Juego en la vin pública } \\
\text { Jueso en el domiclllo }\end{array}$ & $\begin{array}{r}158 \\
69 \\
47\end{array}$ & $\begin{array}{r}24,8 \\
10,9 \\
7,4\end{array}$ \\
\hline $\begin{array}{l}\text { Actividades en sitios públicos } \\
\text { Actividades escolares } \\
\text { Actividades doméstices } \\
\text { Activldades deportlvas }\end{array}$ & $\begin{array}{r}131 \\
91 \\
74 \\
18\end{array}$ & $\begin{array}{r}20,6 \\
14,3 \\
11,6 \\
2,8\end{array}$ \\
\hline Otras & 48 & 7.6 \\
\hline TOTAL & 636 & 100,0 \\
\hline
\end{tabular}

tradomiciliarias aparecen con frecuencia decrecientes. A medida que el niño aumenta en edad disminuye progresivamente la proporción de accidentes derivados de juegos acrecentándose la de actividades escolares. Igualmente el riesgo doméstico, dos a tres veces más importante en la niña que en el varón y que pesa en forma clara en las primeras edades va disminuyendo con los años. A la inversa, las actividades deportivas que aparecen con una frecuencia 6 veces superior en los varones se hacen más riesgosas con el aumento de edad.

Las lesiones producidas se distribuyen en una amplia gama (Tabla No 3 ).

TABLA NO 3

DISTRIBUCLON DE LOSIONES PRODDCIDAS EN 630 ACCLDENTHS EMOLLARTS

\begin{tabular}{|c|c|c|}
\hline Leslones & NQ & \% \\
\hline 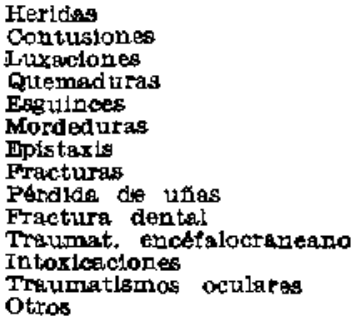 & $\begin{array}{r}342 \\
125 \\
29 \\
38 \\
28 \\
21 \\
18 \\
14 \\
10 \\
6 \\
4 \\
3 \\
2 \\
6\end{array}$ & $\begin{array}{r}53,8 \\
19,7 \\
4,6 \\
4,4 \\
4,4 \\
3,3 \\
2,8 \\
2,2 \\
1,6 \\
0,9 \\
0,6 \\
0,5 \\
0,3 \\
0,9\end{array}$ \\
\hline TOTAI. & 636 & 100,0 \\
\hline
\end{tabular}

La patología cítada en la tabla se ubica anatómicamente en las extremidades inferiores $(30,8 \%)$ cabeza y cara $(28,1 \%)$ extremidades superiores $(26,7 \%)$ siendo menos frecuentes las lesiones generalizadas $(5,6 \%)$ o ubieadas en el tronco $(3,0 \%)$. La Tabla No 4 muestra el tipo de atención recibida.

TABLA NS 4

ATENOION RECLBIDA EN 636 ACCLDENTES FSCOLAFH

\begin{tabular}{|c|c|c|}
\hline TYpo & No & $\%$ \\
\hline $\begin{array}{l}\text { Ningungs } \\
\text { Domiclifo } \\
\text { Botiquin escclar } \\
\text { Médico o dentista privado } \\
\text { Practiosinte } \\
\text { postas de urgenclo } \\
\text { Hospltal } \\
\text { "Compositor" } \\
\text { Cruz Roja }\end{array}$ & $\begin{array}{r}256 \\
128 \\
99 \\
41 \\
30 \\
28 \\
26 \\
17 \\
13\end{array}$ & $\begin{array}{r}40,1 \\
19,9 \\
15,6 \\
6,5 \\
4,9 \\
4,1 \\
4,0 \\
2,8 \\
2,1\end{array}$ \\
\hline TOTAL & 636 & 100,0 \\
\hline
\end{tabular}

Las lesiones registradas son preferentemente heridas y contusiones; ellas se producen con mayor frecuencia en edades bajas y de preferencia en el local escolar a diferencia de las lesiones graves cuyo riesgo aumenta con la edad $y$ se originan principalmente en sitios públicos y en el domicilio. En los niños mayores disminuyen proporcionalmente las lesiones de la cabeza aumentando las de las extremidades. Un $40 \%$ de los casos no requieren o no reciben asistencia profesional lo que probablemente correspon. de a una cierta proporción de accidentes de escasa gravedad (heridas y contusiones de las extremidades) pero puede representar también la ineficacia de los servicios escolares a la despreocupación del medio ambiente frente a pequeñas lesiones.

En nuestra serie un $70 \%$ de los accidentes no determinaron pérdidas de clases (448 casos). Los 188 restantes agruparon 1.761 días con un promedio de 9,4 días. Estimado en forma global los accidentes representan sólo una pequeña proporción (2\%) del ausentismo total, alcanzando en escuelas de riesgo elevado a cifras máximas de $3,2 \%$. EI ausentismo es más frecuente para los accidentes ocurridos en sitios públicos $(37,3 \%)$, siguiendo los escolares $(28,2 \%)$, domiciliarios $(22,9 \%)$ y del tránsito $(11,8 \%)$. 
Ya hemos señalado que en la génesis de los accidentes juegan por una parte condiciones riesgosas ambientales y por otra, factores individuales de mayor susceptibilidad. En nuestra pesquisa las caracteristicas físicas de los Iocales escola. res no mostraron relación con la frecuencia de accidentes, al comparar escuelas con edificio adecuado, existencia de botiquín, etc. con aquellas que no los tenían.

\section{COMFNTARIO}

La investigación practicada no permite señalar la frecuencia exacta de producción de accidentes en el material en estudio, desde el momento que con la técnica empleada (cuestionario a ser llenado por el profesor) se produce un cierto déficit de registro. Hablan en ese sentido las diferencias observadas en diversas escuelas (rango de tasa mensuales fluctuante entre 1,1 y 233,3 por 1.000 ) como igualmente la correlación inversa entre el número de alumnos y la tasa de accidentes. Los datos obtenidos representan así las cifras mínimas del problema. La técnica empleada de modo experimental en esta investigación se ha demostrado así sólo parcialmente útil desde el momento que los resultados sugieren una subenumeración cuyo volumen es imposible de precisar. En todo caso representa un ensayo de interés en la investigación de accidentes no letales, cuya cuantia no ha sido precisada en Chile. Mientras se dispone de adecuada información de muertes y hospitalizaciones por causas violentas, la que ha sido analizada en detalle por nosotros ${ }^{11}$ persisten muchas incógnitas en relación al accidente leve, 100 a 150 veces más frecuentes que el letal, y al domiciliario.

Parece evidente que el volumen que han alcanzado los accidentes no puede seguir ignorándose. Con todas las limitaciones que implica una generalización, si aplicamos la tasa observada en la muestra estudiada por nosotros (2\% mensual) a la población escolar chilena estimada para 1960 en 1.263 .000 niños debería esperarse un total aproximado de 300.000 accidentes anuales, $30 \%$ de los cuales son suficientemente graves como para deter- minar ausentismo y $60 \%$ como para re querir asistencia médica de algún tipo.

La edad escolar representa una época de la vida de especial interés en el problema de los accidentes. Junto a la alta frecuencia y riesgo de violencia aparecen en ella netas posibilidades de acción que se refieren tanto a la mayor seguridad de los locales escolares, en los cuales el niño pasa una importante fracción de su jornada diaria, como a las posibilidades educativas de esta época de gran plasticidad. Sin embargo, los esfuerzos de educación y control no pueden ser indiscriminados; el riesgo es diferente $\mathrm{y}$ proviene de diversas fuentes según la edad que se considere; el énfasis debe ser distinto según se trate de niñas o varones; hay necesidad de cooperación de profesores y maestros y el estudio y solución del problema debería representar una importante proporción de las actividades de los centros de padres, dado el alto volumen de accidentes domiciliarios. Hay igualmente una clara responsabilidad de los médicos escolares, como de las Comisiones Mixtas de Salud y Educación que operan en nuestras áreas hospitalarias.

\section{RESUMEN}

Se presenta la experiencia recogida en accidentes producidos en alumnos de $\mathbf{3 1}$ escuelas primarias de Santiago, investigadas durante los meses de Octubre y Noviembre de 1959 .

Con la técnica empleada se registraron 636 accidentes cuyas características se analizan en detalle.

Destaca el mayor riesgo del niño varón y el incremento de aquel con el aumento de edad. Aparecen como predominantes los accidentes producidos en escuelas y sitios públicos y con menor frecuencia los domiciliarios y del tránsito.

Las lesiones producidas son pricipalmente heridas y contusiones; en un $20 \%$ se registran alteraciones graves; un $40 \%$ de los accidentes no recibieron atención.

En $70 \%$ no determinaron ausentismo escolar; el resto registró una pérdida de clases de 9,4 dias en promedio.

Se comenta la frecuencia y gravedad del problema en Chile y las posibilidades de prevención y control. 


\section{SUMIMARY}

\section{ACCIDENTS IN THE SCHOOL AGE}

Results of an accident research performed in 31 elementary school in Santiago during a two month period of 1959 are presented by the authors.

They describe the main features of 636 accidents emphazising the greater rist. of the boy and the increasing probability of accidents with age. The experience shows school and public accidents as predominant with a lower frequency for the domiciliary and traffic accidents.

Wounds and contusions were registered as the most frequent lesions; $20 \%$ of the accidents had a severe prognosis and $40 \%$ did not receive medical care.

Absenteeism from school averaged 9,4 days; $70 \%$ of the cases did not require physical rest.

Frequency and the severity of the problem in Chile and possibilities of prevention and control are discussed.

\section{ZUSAMMENFASSUNG}

\section{UNFAELLE IM SCHULALTER}

Die Erfahrungen, die bei Unfällen gesammelt wurden, die Schülern von 31 daraufhin in den Monaten Oktober und November 1959 untersuchten Volksschulen Santiagos zustiessen, werden dargelegt.

Mittels der angewandten Methodik wurden 636 Unfälle registriert, deren Besonderheiten in allen Einzelheiten analysiert werden.

Auffallend ist das grössere Risiko der Knaben und dessen Zunahme mit höher werdendem Alter. Als vorherrschend erscheinen die Unfälle, die sich in Schulen und auf Spielplätzen ereigneten; die häuslichen und Verkehrsunfälle sind an Häufigkeit geringer.
Die Schäden, die entstanden, sind hauptsächlich Wunden und Kontusionen; bei $20 \%$ wurden schwere Unfallfolgen verzeichnet; $40 \%$ der Verungluickten benötigten keine Aerztliche Behandlung.

$70 \%$ der Unfälle bedingten kein Fernbleiben von der Schule; beim Rest wurde ein Ausfall von durchschnittlich 9,4 Unterrichtstagen verzeichnet.

Die Häufigkeit der Unfälle und die Schwere des Problems in Chile, sowie die Möglichkeiten der Verhütung und Kontrolle werden erläutert.

\section{BIBLIOGRAFÍA}

1.-ADRIASOLA, G.: ARMIJO, R. Y VENEOAS, F. Algunos hallazgos en encuestas sobre accldentes del tránsito. Comunieselón a la socledad Chllena de Salubridad. 1954 (mimeosraflado).

2.-ADRIASOLA, G.; KAFMMIPFH'TR, A. M.; LEYTON, A.; MAIRA, I, y OPAZO, A. I Toidemlologia de las muertes plolentas. Rev. Chil. Hig. $\mathrm{y}$ Medle. Prev, 14:219, 1952.

3.-ARANDA, L, y MONTOYA, C. - Morbiliad y accldeutes del trebajo en una 1ndugtrls metelúrglca chllena. Rev, S. N. B. 2:69, 1857.

4.-ARAYA, G. - Contribución al estudio de los accidentes, envenenamientos y ylolenclas en is infancla. Tests licenclatura. Escuela de Medictar. Jniversidad de chlle. 1858.

5.-GODDARD, J. L. - La prevenatón de los ace1dentes de la tatancle. Bol. Oflc. Sanit. Pansan. 46:11, 1050 .

6.-HRDALO, B. - Etjologia de los traumatiomas en los accidentes del trabajo. Fl grave problema de los accidentes. Etdit. Dnipersitarla, Santjago de Ch1le. 1859.

7.-JARURET. S. M. - La prevenclón de los aceldentes Industriajes. Fl grape problema de los scctdentes. Edit. Unlversitarla, Eantjaro de Chlle. 1950 .

8.-NAVARBIFTE, O. - Estudia erílco de la legislaclón de accidentes del trabajo. Tesis Eso. Salubridad. 1953 , No 3 .

9. - O. M. S., Grupo Consultito de la. - Ioss accidentes en la Infancia. W. H. O, Tech, Rep, Serles W9 $110,1959$.

10.-PUEHMA, V. - K problema del ausentismo por scoldentés del trabajo en los F'F. CO. del Ertado. Tesis Frac. Bslubridsd, 1953, No 3 .

11.-ROMKRO, H.; VILDOSOLA, J.: MERDINA E. KAEMTFFTER, A. M.; BYMON, F.; MASSAD, F.; JARAMULJO, J. y CRUZAT, S. - Eptdemlología de los accidentes en Chlle. Fil grave problema de los accldentes. Edit. Universitarla, Santlago de Chile, 1059 .

12,-ROMkRO, H. J MORODER. J, - Medlo slglo en la evoluctón de la sanidad $y$ medicina publica en Chile, Santiago, 1957.

I3, -ROSSHLOT, J.: VERA, M. Y BALAZS, O. - Epldemiologia de los eccidentes en la infencla. Pedjatría. 2:47, I959.

14. SWAROOP, S.; ALBRECHT, R, M. IIA GRAB, R. Aocldent mortallts among chlldren. Bull, Wld. Hith. Org. 15:123, 1956. 